This article has been downloaded from IOPscience. Please scroll down to see the full text article. (http://iopscience.iop.org/1478-3975/4/3/004)

More related content is available

Download details:

IP Address: 128.178.66.195

The article was downloaded on 11/12/2007 at 12:59

Please note that terms and conditions apply. 


\title{
A dynamical study of antibody-antigen encounter reactions
}

\author{
Lorenzo Bongini ${ }^{1,6}$, Duccio Fanelli ${ }^{2,6}$, Francesco Piazza ${ }^{3,6}$, \\ Paolo De Los Rios ${ }^{3}$, Michel Sanner ${ }^{4}$ and Ulf Skoglund ${ }^{5}$ \\ ${ }^{1}$ Dipartimento di Fisica, Università di Firenze, V. G. Sansone 1, 50019 Firenze, Italy \\ ${ }^{2}$ Theoretical Physics Group, School of Physics \& Astronomy, University of Manchester, Manchester, UK \\ ${ }^{3}$ Institute of Theoretical Physics, EPFL SB ITP LBS, BSP 720, 1015 Lausanne, Switzerland \\ ${ }^{4}$ SCRIPPS, La Jolla CA 92037, USA \\ ${ }^{5}$ Cell and Molecular Biology Department, Karolinska Institutet, 17171 Stockholm, Sweden \\ E-mail: bongini@fi.infn.it,duccio.fanelli@manchester.ac.uk and Francesco.Piazza@epfl.ch
}

Received 3 July 2007

Accepted for publication 29 August 2007

Published 2 October 2007

Online at stacks.iop.org/PhysBio/4/172

\begin{abstract}
The effects of internal dynamics in diffusion-driven encounters between macro-molecules represent a problem of broad relevance in molecular biology. In this view, we investigate a typical antigen-antibody reaction chain, based on a coarse-grained mechanical model parameterized directly upon results from single-molecule experiments. We demonstrate that the internal dynamics is a crucial factor in the encounter process. To describe our numerical results, we formulate a simple, intuitive theoretical framework, and we develop it analytically. This enables us to show that the inner dynamics of antibody molecules results in a cooperative behavior of their individual sub-units. Along the same lines, we also investigate the case of double binding to multi-valent antigens. Our results quantify the enhancement of avidity afforded by the double binding in excellent agreement with the available experimental data.
\end{abstract}

M This article features online multimedia enhancements

(Some figures in this article are in colour only in the electronic version)

\section{Introduction}

Antibodies are large multi-domain molecules present both in the blood and in many tissues with the purpose of targeting foreign molecules with high specificity, thereby flagging them to different immunological effectors for destruction. Besides being relevant per se to the immunological response system, such binding agents constitute a crucial resource in many contexts, ranging from novel imaging techniques in nanoscience [1] to industrial drug design for cancer therapy [2].

More specifically, antibodies are composed of three subunits - two fragment antigen binding arms (Fabs) connected to a stem $(\mathrm{Fc})$ through a relatively rigid hinge region. Overall, such a structure is extremely flexible so as to enhance its intrinsic capability to bind antigens of various shapes and sizes, from small hormones to large viruses [3, 4]. Molecular

6 These authors contributed equally to the work flexibility implies that the internal dynamics should play a crucial role in regulating the exploration of the many different conformations accessible to the structure [5].

Roughly speaking, the interesting physical processes underlying a typical antibody-antigen reaction are (i) the encounter process (the two partners need to find themselves correctly oriented with the two binding sites exposed to each other) and (ii) the chemical fixation (the necessary chemical bonds need to form at the interaction surface, involving direct and water-mediated hydrogen bonds as well as van der Waals links). Due to the high specificity of antigenantibody reactions, the latter process is much faster than the encounter process, with respect, e.g., to what happens in a typical enzyme-substrate reaction, which makes the overall reaction limited by diffusion [6]. It is therefore interesting to investigate how the internal dynamics of the peculiar threelobe structure of antibodies intervenes in the diffusion-limited encounter process. 


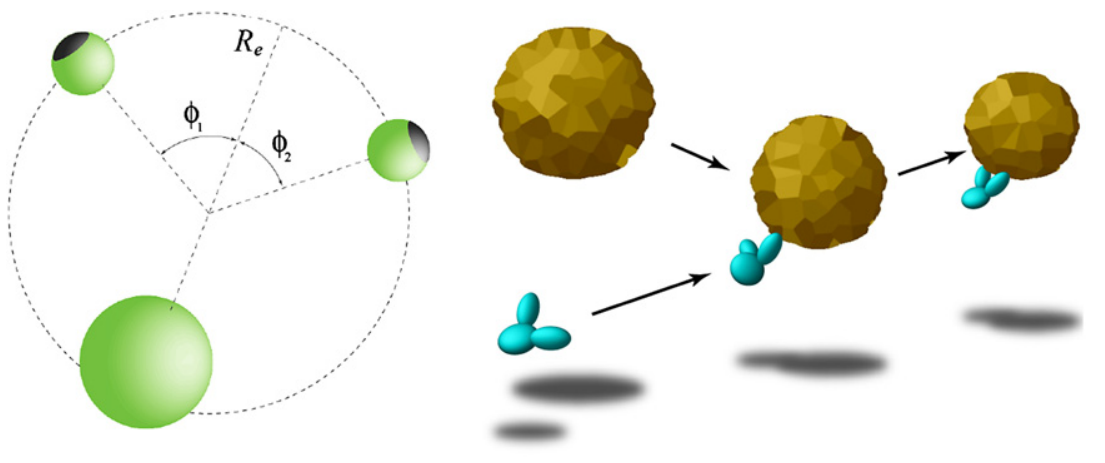

Figure 1. Left panel: three-sphere coarse-grained model of an immunoglobulin. The two smaller spheres represent Fab 1 and 2, while the larger sphere represents the Fc stem. Indicated are the radius $R_{e}$ of the epitope sphere and the Fab-Fc angles $\phi_{1,2}$. Also shown as dark spherical patches are the active regions on the Fabs' tip. The right panel shows a pictorial view of a typical encounter/fixation reaction between an antibody and a relatively large antigen.

In principle, a quantitative theoretical understanding of the dynamical effects in antigen-antibody reactions could be gained through classical all-atom molecular dynamics (MD) simulations [7]. However, the applicability of such a theoretical framework to large systems such as antibody-antigen complexes is severely limited in practice by computational time constraints. Hence, one must resort to coarse-grained models as working tools. In this paper we employ a simple coarse-grained model that represents the antibody structure as a collection of three rigid bodies hinged at a common point and moving under the action of a potential energy that is parametrized directly from singlemolecule experiments [8]. Based on this formulation, we here simulate the Brownian dynamics of an antibody to inspect the entire pathway of a typical antibody-antigen encounter reaction (pictorially illustrated in figure 1).

In the first part of the paper, we measure the antibodyantigen encounter rate for small isotropic antigens of different sizes, roughly up to the breadth of an antibody. In the standard theory of diffusion-limited encounter reactions between agents with no internal dynamics, the rate is uniquely specified by the encounter geometry, such as the size and orientation of binding sites. Overall, a given binder is characterized by a unique, constant steric factor. We find that this simple picture no longer holds for an antibody, as a result of its internal dynamics, resulting in a steric factor which depends on the antigen size. We rationalize this finding within an intuitive model that combines the internal dynamics with the basics of the theory of encounters between anisotropic static objects. Our theory allows us to derive a formula that measures the degree of interference between the two Fabs in the encounter process with antigens. We find that for small antigens the antibody is a less effective agent with respect to two isolated identical Fabs in solution (negative cooperativity). Interestingly for larger antigens, but still such that each Fab binds individually to separate objects, we find a transition to a state of constructive interference (positive cooperativity), where the antibody takes advantage from its internal structure and dynamics with respect to two isolated Fab agents.

We turn next to examine the encounter with antigens much larger than the antibody, such as big viruses. In this case we focus on the role of the internal dynamics in the avidity, i.e. the modulation of the encounter of the second Fab once the first Fab-virus contact has been established. Our simplified theoretical framework enables us to show that the binding of the second Fab arm to the same antigen is indeed more favorable than the binding of the first arm. Moreover, we are also able to quantify this effect, thus improving on previous models [9] and approaching more closely the experimental results [10].

\section{Methods}

We simulate the Langevin dynamics of one antibody and $N_{a}$ antigens at room temperature. The antibody is modeled as consisting of three units (two Fabs and one Fc) joined at a common point and interacting according to the force-field computed directly from single-molecule cryo-ET experiments in [8] (see figure 1). The Fabs and the Fc are approximated by impenetrable effective spheres of radii $R_{1}=16 \AA$ and $R_{2}=34 \AA$, respectively. The values of $R_{1}$ and $R_{2}$ have been chosen so that Fab-Fab and Fab-Fc angles at the contact position would reproduce the experimental values reported in [8]. The friction coefficients have been set so as to reproduce the experimental values of the $\mathrm{Fab}$ and $\mathrm{Fc}$ diffusion coefficients in water $[11,18]$. The antigens, described as non-interacting, are also represented as spherical particles of variable radius.

All particles are confined in a spherical box of radius $L$ with reflecting boundary conditions. The capture occurs when an antigen reaches one of the Fabs in its reactive region, modeled as a circular patch on its outer surface. Each time an antigen is absorbed, it is replaced by another particle randomly positioned at a distance $L$ from the center. This amounts to imposing that the flux of incoming particles be equal to the flux of particles leaving the box, as required by the condition of stationarity.

Numerical simulations are performed in a reference system diffusing with the antibody. This amounts to replacing the antigen diffusion coefficient $D_{a}$ with $D_{e}+D_{a}$, where $D_{e}=k_{\mathrm{B}} T / \gamma_{e}$ is the diffusion coefficient of the epitope sphere. The stochastic components of the forces are set accordingly. The simulations presented in this paper refer to $L=600 \AA$ and $N_{a}=50$.

The binding of the second Fab is also subject to numerical investigation. For this purpose, the outer edge of the bound Fab 
is made to coincide with the active patch localized on an ideally planar antigen. This amounts to considering large antigens, e.g. viruses or cells. Equivalently, it refers to the case where antigens are immobilized on a planar interface, a configuration of paramount importance in many experimental applications with functionalized surfaces. The antibody evolves under the effect of this additional constraint. We model the adjacent antigenic site as a circular disc of radius $a$, randomly positioned on the plane, within a distance of $2 R_{e}$ from the first binding site. This corresponds to a surface concentration of active patches $[A]_{s}=1 /\left(4 \pi R_{e}^{2}\right)$. The second Fab explores the surrounding space and will eventually fall onto the plane with its tip within the available active site. Our simulations show that the probability of staying outside the designed area of interaction decays exponentially, with an average entry time $\tau$. The latter provides a measure of the average time needed for the second $\mathrm{Fab}$ to reach the binding area. We find $\tau^{-1} \simeq 10^{-8} \mathrm{~ns}^{-1}$ for $a=5 \AA$.

\section{Results}

Our objective is to measure the encounter rate for antigens of different sizes. As a first task, we shall focus on the case of small to intermediate antigens in order to pinpoint the relevant dynamical phenomena involved in the encounter process. Furthermore, we shall concentrate on the encounter rate of a whole antibody as compared to that characterizing a single Fab unit for the same antigen, in the effort to rationalize and quantify the combined action of the two Fab arms within the same structure. Subsequently, we shall turn to investigate the case of double binding to large, multi-valent antigens with the aim of elucidating the role of dynamics in the intriguing property of antibodies termed avidity (see the glossary).

\subsection{Antibodies looking for small antigens: binding the first Fab}

We model an antibody as an ensemble of three spheres constrained to move à la Langevin at a fixed distance from a common hinge point under the action of the internal angular forces derived from cryo-electron experiments in [8] (see figure 1). Antigens are represented by spherical particles diffusing freely in a spherical bounding box with reflecting and constant-flux boundary conditions (see section 2).

In order to investigate the role of the antibody internal dynamics we need to identify a reference static case. For this purpose, antigens may be treated as spherical particles of radius $r$, while the immunoglobulin molecule can also be identified with a sphere. The most natural choice is the sphere that corresponds to the Stokes radius of an antibody as determined from its diffusion coefficient [11], that is $64 \AA$. We shall call this the epitope sphere since it well approximates the surface spanned by the motion of the Fab tips (see figure 1). Within this simplified scenario, the encounter rate is given by the well-known Smoluchowski formula [12]

$$
\kappa_{S}=4 \pi c_{\infty}\left(D_{a}+D_{e}\right)\left(r+R_{e}\right),
$$

where $D_{a}$ and $D_{e}$ denote the diffusion coefficients of the antigen and of the epitope sphere, respectively, whereas $c_{\infty}$ is the bulk antigen concentration.

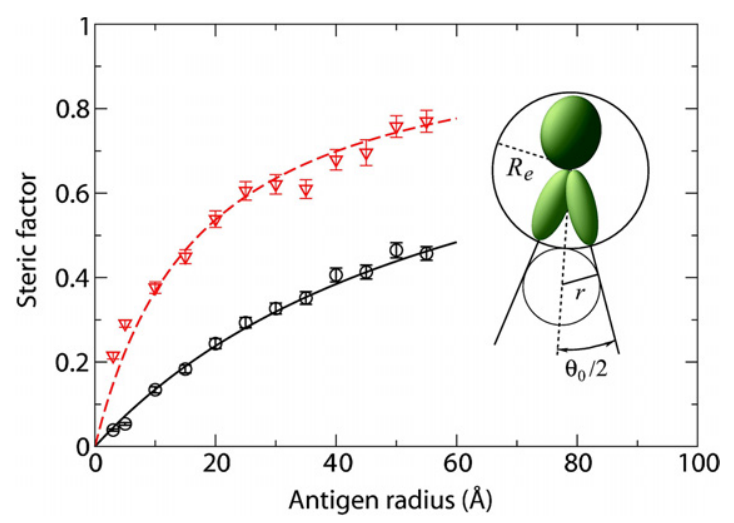

Figure 2. Steric factor as calculated according to formula (2) from the numerics for different sizes of the active surface fraction $\sigma$ on the two Fabs. Circles: $\sigma=0.02$. Triangles: $\sigma=0.34$. The solid lines refer to the theoretical profile (3) with $\alpha=6.4$, as determined numerically. Here the bulk antigen concentration is fixed by normalizing to one the distribution of the antigen-antibody distance within the spherical simulation box. The drawing represents a sketch of the encounter cone analogy.

An encounter event occurs when an antigen reaches one of the two Fabs in its reactive region, namely a circular patch on the Fab sphere of given surface fraction $\sigma$ (see figure 1). Correspondingly, the encounter rate $\kappa$ for a given value of $r$ can be estimated numerically by following many trajectories of the combined antigen-antibody system and calculating the inverse of the average encounter time (see section 2). An effective steric factor $f$ can then be measured, quantifying the antibody's loss of encounter effectiveness with respect to the Smoluchowski approximation

$$
f=\frac{\kappa}{\kappa_{S}} \text {. }
$$

Results of our numerical study are reported in figure 2, where $f$ is plotted as a function of the antigen size. Simulations were performed for $\sigma$ ranging from 0.02 to 0.34 , which correspond to spanning a range of active surfaces from $60 \AA^{2}$ to $1100 \AA^{2}$.

It should be emphasized that the buried surface area on both partners in a typical antigen-antibody complex seems to vary with the antigen size. In particular, from crystallographic data $\sigma$ is seen to increase roughly linearly with the antigen radius for small antigens (smaller than about $10 \AA$ ), whereas it is almost constant and never larger than about $1200 \AA^{2}$ for larger antigens [13]. For the sake of simplicity, in our simulations we kept $\sigma$ fixed, selecting values in the whole observed interval. We did not get qualitatively different results at different values of $\sigma$. Had we included the effect of varying $\sigma$, we would have recovered the same trend as in figure 2, but for a steeper increase in the small antigen region.

In the crudest approximation the antibody may be pictured as a rigid sphere of radius $R_{e}$, with an active surface equal to twice the extension of a Fab's tip. In diffusion-driven reactions between two spherical species, one chemically isotropic and the other characterized by a localized reactive site, the steric factor $f$ does not depend on the radius of the target isotropic particle and is well approximated by the square root of the active surface fraction on the anisotropic sphere [14, 15] (see also the appendix). According to this simplified description, 
the steric factor should be $f=\sqrt{2 \sigma}\left(R_{1} / R_{e}\right)$, where $R_{1}$ is the radius of the Fab sphere. Accordingly, $f$ should be in the range $f=0.05-0.2$ for the considered interval of $\sigma$. The results reported in figure 2 show that the measured encounter rate is not a constant and increases with the antigen size well beyond the above estimate, showing to saturate for larger and larger antigens. Such a scenario is reminiscent of reactions between pairs of species where the binding site of one moiety is hidden in a pocket whose accessibility strongly depends on the steric hindrance of the other one. However, this is certainly not our case. Our results are a direct consequence of the antibody internal dynamics.

Intuitively, we can rationalize the results reported in figure 2 in the following fashion. For small antigens of linear size up to about $10 \AA$ (say a hormone molecule), the great degree of flexibility of the two Fabs is of little help in capturing small diffusing objects, so the steric factor is small and dominated by the geometric effects (namely the limited extension of the active regions on the Fab tips). However, as the antigens get larger and larger, the internal dynamics of the Fab pair starts making a difference. Overall, the antibody is now encountering much more than its geometric static analog, namely the epitope sphere with two fixed active patches. This regime concerns antigens of intermediate size, i.e. macromolecules of diameter comparable to that of a single $\mathrm{Fab}$, that is of the order of $100 \AA$. Eventually, for extremely large antigens, of the size of a whole antibody and larger, such as viruses, the steric factor has no choice but to converge to a fixed estimate.

In order to decipher quantitatively the mechanism underlying our numerical observations, we can reason as follows. It is clear that the necessary condition for the encounter to occur is that the antigen and the antibody find themselves correctly oriented when close enough to each other. This amounts to saying that one of the two Fab tips should find itself in correspondence to the incoming antigen, i.e. inside an encounter cone of angular aperture $\theta_{0}$. The latter may be thought of as the angle under which an antigen is seen from the center of the antibody at the contact distance, which defines a variable surface portion of the epitope sphere $\Delta S_{e} / S_{e}$ (see the sketch in figure 2). However, it can be argued that such an effective aperture depends on the finite extension of the active patch on the Fab's tip, narrower apertures corresponding to smaller patches. This effect can be modeled by assigning to the incoming antigen an effective size $\rho \propto r$, which also depends on $\sigma$. From our simulations we find that $f \propto r$ for small antigen radii. Since, for increasingly small values of $\sigma$, $f$ vanishes as $\sqrt{\sigma}$ for arbitrary values of $r[14,15]$, we assume $\rho=\alpha r \sqrt{\sigma}$, where $\alpha$ is a constant factor.

Hence, considering the contribution of the two Fabs to the encounter rate to be additive, i.e. assuming for simplicity that the two active tips move in an uncorrelated fashion while exploring the epitope sphere, for small encounter cones one can write $[14,15]$

$$
f=2 \sqrt{\frac{\Delta S_{e}}{S_{e}}}=2 \sin \left(\frac{\theta_{0}}{4}\right) \approx \sin \left(\frac{\theta_{0}}{2}\right)=\frac{\alpha r \sqrt{\sigma}}{\alpha r \sqrt{\sigma}+R_{e}},
$$

where we have also assumed that the encounter cone is not large. To be more precise, for $\theta_{0}<\pi / 4$, the error made in deriving the approximate formula (3) is less than $8 \%$.

The excellent predictive adequacy of our simple analytical estimate is clearly demonstrated in figure 2 through comparison with the numerics. The agreement is remarkably good for both values of $\sigma$ and, despite the approximations introduced, also for relatively large values of $r$. Equation (3) predicts that the steric factor asymptotically reaches the value $f=1$ for large antigen radii, meaning that the internal dynamics has become infinitely fast with respect to the diffusion of the target. Such a (formal) limit can be imagined to describe the recurrent experimental setting where the antigens are attached onto a planar interface. However, it is fair to assume that the precise limiting value should be somewhat lower than $f=1$ and that our asymptotic result is the consequence of slightly overestimating the effects of Fab's dynamics.

It is interesting to exploit the theoretical framework outlined above in the case of a chemically anisotropic antigen, by translating the information contained in equation (3) in terms of an entropic barrier. This can be done by recalling that the entropy change associated with the loss of rotational freedom reads [16]

$$
\Delta S_{\mathrm{rot}}=-k_{B} T \log \left(\sqrt{\sigma_{a}} f\right),
$$

where we have introduced the fraction of reacting surface on antigens $\sigma_{a}$. Assuming that the active patch is of the same size in both species, e.g. of the order of $1000 \AA^{2}$, the above expression gives $\Delta S_{\text {rot }}>1-1.5 \mathrm{kcal} \mathrm{mol}^{-1}$ for antigens of linear size greater than about $40 \AA$. For antigens of intermediate size, that estimate reproduces at most an entropic cost of about $15-20 \%$ of the experimental data, which is around $7 \mathrm{kcal} \mathrm{mol}^{-1}$ [16]. To account for the whole entropic cost would of course require including the contribution of other effects, such as translational entropy or vibrational entropy costs associated with establishing a stiffer complex and reduction of solventaccessible volume.

3.1.1. Cooperativity effects. The above sketched framework can be employed to address an important issue of immunoglobulin-binding kinetics, namely the degree of interference displayed by the two identical active units (the Fabs) as far as their individual encounter rates are concerned. In other words, antibodies are provided with two identical binding units that could in principle contribute in a simple additive way to the overall encounter rate, or show some degree of positive (cooperativity) or negative (anti-cooperativity) interference.

In [17] we investigated a simplified analytical model of the antigen-antibody encounter that predicts anti-cooperative behavior, the system being closer and closer to the additive case the farther apart the two Fabs. However, the model of [17] has two major limitations. First, it is strictly valid only for small antigens-say of the size of small hormone molecules. Second, it neglects the presence of the Fc fragment as an additional, totally reflecting unit. Indeed, the present realistic numerical inspection of the encounter process allows us to 


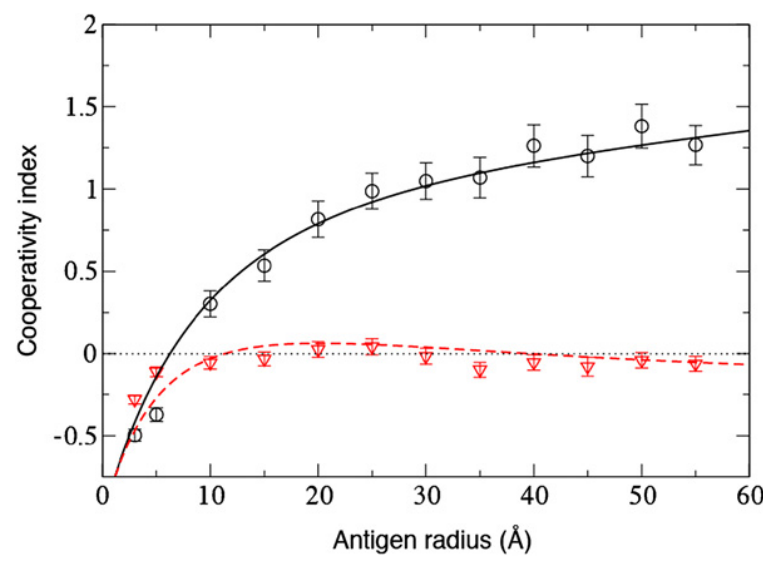

Figure 3. Cooperativity index (5) as a function of the antigen radius for different sizes of the surface fraction $\sigma$ on the two Fabs. Circles: $\sigma=0.02$. Triangles: $\sigma=0.34$. The solid and dashed lines are plots of the analytical estimate (7) with $\alpha=6.4$. All data have been rescaled in order to compensate for the presence of a finite bounding box in the simulations.

quantify to a greater detail the two-body correlations within the antibody.

As a quantitative measure of the cooperative effect, we introduce the following indicator:

$$
\mathcal{I}=\frac{\kappa}{2 \kappa_{1}}-1 \text {, }
$$

where $\kappa_{1}$ is the rate associated with a single Fab diffusing in solution, modeled as an anisotropic sphere of radius $R_{1}$ with the same active surface fraction $\sigma$ as within the antibody. By definition, $f=\kappa / \kappa_{S}$. Consequently, expression (5) can be rewritten as

$$
\mathcal{I}=\frac{f}{2 \sqrt{\sigma}\left(\kappa_{S 1} / \kappa_{S}\right)}-1,
$$

where we have introduced the Smoluchowski rate $\kappa_{S 1}$ that characterizes encounters with a single, fully-reactive Fab through the approximate relation $\kappa_{1}=\sqrt{\sigma} \kappa_{S 1}$. Combining definition (1) and expression (6), and recalling equation (3), we obtain

$$
\mathcal{I}(r)=\frac{D_{e}}{2 D_{1}}\left(\frac{\alpha r}{\alpha r \sqrt{\sigma}+R_{e}}\right) \frac{\left(r+R_{e}\right)^{2}}{\left(r+R_{1}^{d}\right)\left(r+R_{1}\right)}-1,
$$

where $D_{1}=k_{B} T / 6 \pi \eta R_{1}^{d}$ is the translational diffusion coefficient of a single spherical Fab, $R_{1}^{d} \approx 31 \AA$ being its diffusive radius $[11,18]$.

The results are displayed in figure 3 where $\mathcal{I}(r)$ is plotted as a function of the antigen radius $r$ for the aforementioned values of $\sigma$. We see that the extension of the active region on the Fabs' tip controls the degree of positive interference characterizing the combined action of the two units. Nevertheless, anti-cooperativity is always found for small antigen radii, an observation that nicely fits into the scenario proposed in [17]. Surprisingly, when increasing the antigen size, the antibody enters a region where the two Fab units behave cooperatively, thus suggesting an interesting interpretative framework for rationalizing the double-armed structure common to all antibodies.

For larger antigens, a second transition is observed and anti-cooperative behavior eventually recovered. It is

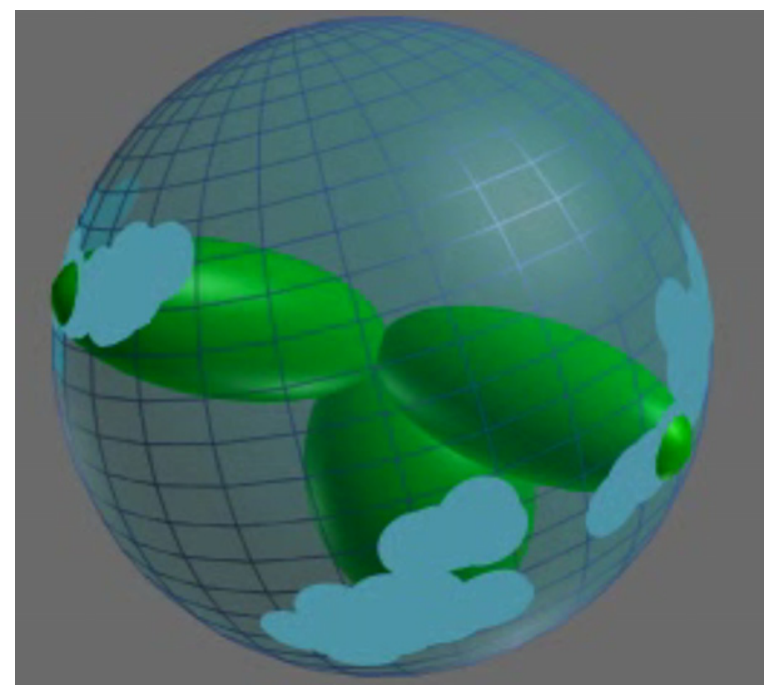

Figure 4. Representative frame of the movie annexed to the paper as supplementary online material available from stacks.iop.org/PhysBio/4/172. The animation shows a trajectory of an antibody molecule as calculated from our coarse-grained model. The surface spanned by the Fab tips on the epitope sphere is shown for some time after disappearing.

interesting to note that it is precisely to large antigens that an antibody binds with both arms on the same target, thus remarkably increasing the overall binding strength. Hence, in the large antigen region antibodies would find the justification of their double-armed structure in the substantial gain in binding solidity that largely compensates the unavoidable moderate anti-cooperativity. More precisely, when $\sigma=0.34$ (large patches on the Fabs'tips), the transition occurs at $r \simeq$ $40 \AA$, a value that falls in the explored region of parameters (see dashed line in figure 3). Conversely, for $\sigma=0.02$ (smaller patches) formula (7) predicts $r \simeq 400 \AA$. This estimate ultimately relies on equation (3), and the comments on the validity of its extrapolation to large values of $r$ still apply. A slightly lower value of the transition radius might hence be expected when performing direct numerical simulations.

As a final remark let us note that we are in a position to quantitatively compare our prediction to the cooperativity index as deduced from the ratio of experimental binding rates measured for the antibody-antigen and Fab-antigen systems, respectively. In fact, due to the limited spatial range of the forces determining the chemical fixation of a given pair, binding rates are well approximated by the product of the encounter rates and a constant that depends on the free energy contribution of the site-epitope interaction. Consequently, the ratio between the two rates is uniquely determined by the diffusion-limited part of the process and can be therefore correctly interpreted within our model, which at present does not incorporate the short-range interactions that stabilize the encounter complex.

Usually, the kinetics of binding of antibodies and Fab fragments to their antigens is investigated through measurements at the interface, e.g. Biacore [19] and Resonant Mirror Biosensors [20]. The experiments reported in $[21,22]$ both point to an anti-cooperative behavior, although 
the binding rates reported therein lead to different cooperative indexes falling in the $[-0.54,-0.4]$ and $[-0.2,-0.07]$ ranges, respectively. These findings are compatible with the anticooperativity effect that we detect for large antigens, since, formally, the experiments at the interface correspond to the limit of the infinite antigen size.

Finally, we would also like to stress that in principle a reliable experimental measure of anti-cooperativity in solution would allow one to estimate the size of the active surface on the Fab tip. This is a very elusive quantity that up to now has been estimated only by measuring the contact surface of already stabilized complexes by means of x-ray crystallography [13]. Nevertheless, the same quantity may vary in the transition state between the bound and unbound configurations. By inverting formula (7) with respect to $\sigma$ one could estimate its value, based on kinetic data.

\subsection{Double binding to multi-valent antigens}

Let us turn now to investigate the case of double binding to large, multi-valent antigens. Immunoglobulins are bivalent structures, and are therefore capable of binding two sites on the same antigen under favorable conditions. Importantly, multi-valent interactions may achieve higher binding affinity, usually termed avidity, to underline the augmented strength of the interaction with respect to the sum of the individual binding strengths of each Fab. Remarkably, the case of multiple binding events to multi-valent antigens will allow us to draw a quantitative comparison with experimental measurements.

The process of monovalent adsorption is characterized by the equilibrium constant $K_{S}=k_{S} / k_{-S}$, while the conversion from mono-valent to bi-valent is controlled by $K_{D}=k_{D} / k_{-D}$. The labels $S$ and $D$ are introduced to indicate single and double binding, respectively. As a first approximation, the binding rates $k_{S}$ and $k_{D}$ can be estimated as the product of the encounter rates, $\kappa_{S}$ and $\kappa_{D}$, respectively, and the associated reaction probabilities for the encounter complex to be stabilized. We assume the latter to be identical, thus neglecting higher order thermodynamics corrections. Furthermore, we assume that $k_{-S} \approx k_{-D}$. Under these assumptions $K_{D} / K_{S}=\kappa_{D} / \kappa_{S}$.

We can limit our analysis to the case where the antigens are immobilized on a planar surface (or equivalently to the case of a large virus antigen), and hence fix $f=1$ in equation (2). This amounts to assuming a fast internal Fab dynamics, thus representing the antibody as a fully absorbing sphere. In this limit, existing estimates $[24,25]$ can be applied in order to evaluate $\kappa_{s}$, which corresponds to the rate of encounter with one of the many equivalent binding epitopes on the antigen surface. Approximating such patches with circles of radius $a$ and calling $[A]_{s}$ their surface density one can show that

$$
\kappa_{S}=D_{e} a[A]_{s},
$$

where $a$ is the radius of a perfectly absorbing circular disc and $[A]_{S}$ represents the surface concentration of the active patches.

Once the first Fab has reached the antigen active site, the system experiences a reduction in mobility, the outer edge of the bound arm being constrained in correspondence with the target. The second Fab explores the surrounding space looking for an additional connection to be established. Numerically, one can calculate the average time $\tau$ needed for the second Fab to enter a circular discs of radius $a$ localized at the planar interface, for a given value of $[A]_{s}$. Then $\kappa_{D} \approx 1 / \tau$ and therefore,

$$
\frac{K_{D}}{K_{S}} \approx \frac{4 \pi R_{e}^{2}}{D_{e} a} \frac{1}{\tau}
$$

where we have used the expression $[A]_{s}=1 /\left(4 \pi R_{e}^{2}\right)$ (see section 2). Numerical simulations performed for the case $a=5 \AA$ gives $K_{D} / K_{S}$ of the order $10^{-4} \AA^{-1}$, corresponding to a positively cooperative binding effect. This result is in excellent agreement with the measurements reported in [10], where an innovative microfluidic strategy complemented with total internal reflection microscopy is employed. Success in reproducing the correct order of magnitude provides an a posteriori validation of our theoretical picture. In this respect, it is worth recalling that the widely accepted model by Crothers and Metzger [9] predicts, in the case of antigens absorbed on a planar surface, a ratio $K_{D} / K_{S} \approx$ $R_{e}^{-1} \approx 0.015 \AA^{-1}$ [23], well above the experimentally measured value.

\section{Conclusion and outlook}

In this work we employ the mechanical model of antibodies worked out in [8] from Cryo-ET experiments in order to investigate the antigen-antibody encounter process. Our results indicate that antibodies can be operatively regarded as anisotropic spheres, characterized by an effective reactive region that depends on the size of the antigens and on the extension of the active regions on the Fabs' tips. Based on this analogy, we find that the encounter reaction largely proceeds in a cooperative manner with respect to the case of two isolated Fabs. Furthermore, we quantify these effects analytically along the reaction pathway.

Our analysis shows that simple, intuitive arguments may be successfully employed to construct theoretical interpretative frameworks of complex dynamical phenomena. This entails the possibility of introducing elementary analytical expressions able to capture the core of biomolecular functional machineries. This is clearly demonstrated by our analysis of single and double binding of immunoglobulins to multi-valent antigens. We are able to quantify the effects of cooperativity within a simple theoretical framework whose predictions correlate quantitatively with the available experimental measurements.

Overall, our analysis clearly shows that the experimentally observed behavior of a large and complex structure such as an antibody strongly depends on its flexibility and on its dynamics. Moreover, we show that the latter can be analyzed by using an extremely coarse-grained scheme, whose predictions compare favorably with experiments and provide a clear and intuitive new perspective to look at the biological functions of biological macro-molecules.

As a final remark, we stress that the physical input to our mechanical model comes directly from singlemolecule experiments. Besides Cryo-ET [26], there is an ample spectrum of techniques that may likewise supply the 
required knowledge. Promising examples include singlepair fluorescence resonance energy transfer (spFRET) and single-molecule fluorescence polarization anisotropy (smFPA) [27]. In actual fact, our scheme may be thought of as an utterly general strategy to study protein dynamics through experimentally aided computer simulations.

As a visual example of our results, we annex to this paper a movie of the antibody dynamics from our simulations as supplemental material available from stacks.iop.org/PhysBio/4/172. The surface spanned by the Fab tips on the epitope sphere is explicitly drawn and made to persist for some time in order to provide a pictorial representation of the Fabs' ability to cover the epitope surface.

\section{Acknowledgments}

This work was supported by grants from the Agouron Institute, the Karolinska Foundation, Sidec Technologies AB, the Foundation of Knowledge and Competence Development, the Swedish Foundation for Strategic Research, the Swedish Research Council and by support from the EU grant to a PF6 NoE 3D-EM. LB was also the holder of a special grant from the Swedish Institute (Italy). Note that US is founder of Sidec Technologies AB, Sweden, which holds the rights for one of the softwares used in this study. However, COMET image processing is available for academic research through US, and commercially through Sidec Technologies AB. The authors are grateful to Sara Sandin for providing her data and for interesting discussions.

\section{Appendix. Diffusion-controlled reaction between anisotropic species}

Consider the general problem of an encounter between two species $A$ and $B$ with concentration $\rho_{A}$ and $\rho_{B}$, respectively. The rate of change of the concentration of the encounter complex $A B$ is given by

$$
\frac{\mathrm{d} \rho_{A B}}{\mathrm{~d} t}=k \rho_{A} \rho_{B}-k^{\prime} \rho_{A B},
$$

where $k$ is the encounter rate constant and $k^{\prime}$ is the decay (or fixation) rate of the encounter complex. The simplest approximate calculation of $k$ was performed by Smoluchowski in 1916 under the hypothesis of chemically isotropic reactants [12]. By treating the two reacting species $A$ and $B$ as spheres of radius $R_{1}$ and $R_{2}$ with reactivity homogeneously distributed over their surface, one obtains the so-called Smoluchowski encounter rate constant

$$
k_{S}=4 \pi D R,
$$

where $D=D_{1}+D_{2}$ is the coefficient of relative diffusion and $R=R_{1}+R_{2}$ is the encounter distance.

In general, however, the diffusion-controlled reaction (A.1) often displays chemically anisotropic behavior: there is only a restricted active region on the surface of the reactants where the reaction can occur. The reduction of encounter effectiveness caused by localization of the reactive sites with respect to the ideal Smoluchowski setting can be described by introducing a steric factor $f$ through the relation:

$$
k=f k_{s} .
$$

Numerous theoretical works report the computation of the steric factor $f$ for different classes of diffusion-controlled reactions between anisotropic agents. Mathematically, such reactions are described by mixed-boundary value problems, that can be solved only in some specific cases. Traytak considered the case of a diffusion-controlled reaction between a chemically isotropic sphere and a chemically anisotropic one, carrying an axially symmetric circular absorbing patch [14]. He was able to obtain an exact solution of this model by using the formalism of dual series relations (DSR). An infinite set of linear equations is constructed and solved iteratively after appropriate truncation. The zeroth-order approximation leads to

$$
f^{(0)}=\frac{1}{2 \pi}\left(\theta_{0}+\sin \theta_{0}\right),
$$

where $\theta_{0}$ labels the angular aperture of the localized active site. Note that this expression was also derived in [28]. The firstand second-order approximation read, respectively,

$$
\begin{gathered}
f^{(1)}=\frac{f^{(0)}}{1-f^{(0)}} \\
f^{(2)}=\alpha f^{(1)},
\end{gathered}
$$

where

$$
\alpha=\frac{1+\omega}{1-f^{(1)} \omega},
$$

and

$$
\omega=\frac{\sin ^{2} \theta_{0}\left(1+\cos \theta_{0}\right)^{2}}{\left(\theta_{0}+\sin \theta_{0}\right)\left[4 \pi-\left(\theta_{0}+\frac{1}{3} \sin 3 \theta_{0}\right)\right]} .
$$

The above second-order approximation (A.8) turns out to yield an extremely accurate estimate of the exact encounter steric factor. Surprisingly enough, remarkably good agreement is also found with the simple formula

$$
f=\sqrt{\frac{\Delta S}{S}}
$$

where $\Delta S / S$ represents the absorbing surface fraction of the anisotropic sphere. Hence, it is established that the encounter rate scales as the linear dimension of the absorbing patch. The above discussion applies for non-rotating agents: the theory can however be extended so as to incorporate the effect of rotation. In the case of small active patches, one can show that the steric factor is still proportional to the square root of the active surface fraction. This issue is extensively discussed by Shushin and Barzykin [15].

Other pieces of work characterize the bimolecular diffusion-controlled reaction in the case where both reacting molecules are anisotropic. Quite accurate formulae are derived in the closure (constant flux) approximation [29, 30]. Simpler expressions are obtained by assuming the quasi-chemical hypothesis [31, 32]. Also in this case, however, numerical calculations (not reported here) clearly indicate that the closed analytical relations for the steric factor are well interpolated by the following generalization of equation (A.9):

$$
f=\sqrt{\frac{\Delta S_{A}}{S_{A}} \frac{\Delta S_{B}}{S_{B}}} .
$$




\section{Glossary}

Antibodies. Antibodies, also known as immunoglobulins (Ig), are large three-domain proteins present in the blood in vertebrates with the function of binding to foreign agents (antigens) causing potential harm to the organism. Antibodies can bind to anything from small molecules of a few atoms (haptens) to large viruses and cells. There are several different kinds of antibodies, which are grouped into different families such as $\operatorname{IgG}, \operatorname{IgM}$ and $\operatorname{IgA}$. For example, five different antibody isotypes are known in mammals. Although the general structure of all antibodies is very similar, two identical small regions at the tips of the two smaller domains are extremely variable in sequence (epitopes). Thus, millions of antibodies exist that differ only in those small regions, each variant binding specifically to a unique target. In this way, the immune system is able to recognize an equally wide diversity of antigens.

Diffusion-limited reactions. Consider a chemical reaction of the kind $A+B \rightarrow A B \rightarrow P$, that describes the formation of a product $(P)$ upon stabilization of an encounter complex $(A B)$, formed when two chemical species $A$ and $B$ get in contact. In the gas phase the rate of approach is fast, and hence the encounter complex forms at a rate that is comparable if not faster than the rate of chemical fixation regulating the reaction $A B \rightarrow P$. Conversely, for reactions taking place in the liquid phase, the formation of the encounter complex is a diffusive process and hence it is normally slower than the rate of stabilization of the intermediate complex, which in solution usually involves both the formation of covalent and hydrogen bonds at the interface between the two ligands. In this case, the reaction is said to be limited by diffusion.

Cooperativity. Let us consider a process where a given observable measures the combined activity of many identical agents individually performing the same action. The overall process is said to be positively cooperative (negatively cooperative or also anti-cooperative) if the combined measure is greater (lower) than the sum of the individual measures. For example, in the case of a reaction between a multi-domain molecule and a given agent, the reaction will proceed cooperatively if the rate exceeds the sum of the rates measured for the individual reactions between the same agent and each domain.

Avidity. Avidity is the affinity of antibodies binding to multivalent antigen molecules. Avidity strengthens binding to antigens with multiple identical epitopes by double binding of a single Ig molecule at the antigen surface. This is the reason why antibodies polymerize in order to bind more effectively very large antigens - the more antigen-binding sites bound to Igs, the higher the overall avidity for antigen. This feature of antibodies is also termed cross-linking and is crucial for the functioning of the immune system.

\section{References}

[1] Raab A, Han W, Badt D, Smith-Gill S J, Lindsay S M, Schindler H and Hinterdorfer P 1999 Antibody recognition imaging by force microscopy Nat. Biotechnol. 17902

[2] Milenic D E, Brady E D and Brechbiel M W 2004 Antibody-targeted radiation cancer therapy Nat. Rev. Drug Discovery 3488

[3] Roux K 1999 Immunoglobulin structure and function as revealed by electron microscopy Int. Arch. Allergy Immunol. 120 85-99

[4] Nezlin R 1990 Internal movements in immunoglobulin molecules Adv. Immunol. 48 1-40

[5] McCammon J A and Karplus M 1977 Internal motions of antibody molecules Nature 268 765-6

[6] Raman C S, Jemmerson R, Nall B T and Allen M J 1992 Diffusion-limited rates for monoclonal antibody binding to cytochrome c Biochemistry 31 10370-9

[7] Brooks B R, Bruccoleri R E, Olafson B D, States D J, Swaminathan S and Karplus M 1983 CHARMM: a program for macromolecular energy, minimization, and dynamics calculations J. Comput. Chem. 4 187-217

van Gunsteren W F and Berendsen H J C 1990 Computer simulation of molecular dynamics: methodology, applications, and perspectives in chemistry Angew. Chem. Int. Edn Engl. 29 992-1023

Pearlman D A, Case D A, Caldwell J W, Ross W R, Cheatham T E III, DeBolt S, Ferguson D, Seibel G and Kollman P 1995 AMBER, a package of computer programs for applying molecular mechanics, normal mode analysis, molecular dynamics and free energy calculations to simulate the structural and energetic properties of molecules Comput. Phys. Commun. 91 1-41

[8] Bongini L, Fanelli D, Piazza F, De Los Rios P, Sandin S and Skoglund U 2004 Freezing immunoglobulins to see them move Proc. Natl Acad. Sci. USA 101 6466-71

Bongini L, Fanelli D, Piazza F, De Los Rios P, Sandin S and Skoglund U 2005 Dynamics of antibodies from cryo-electron tomography Biophys. Chem. 115 235-40

[9] Crothers D M and Metzger H 1972 The influence of polyvalency on the binding properties of antibodies Immunochemistry 9 341-57

[10] Yang T, Baryshnikova O K, Mao H, Holden M H and Cremer P S 2003 Investigations of bivalent antibody binding on fluid-supported phospholipid membranes: the effect of hapten density J. Am. Chem. Soc. 125 4779-84

[11] Carrasco B, De la Torre J G, Byron O, King D, Walters C, Jones S and Harding S 1999 Novel size-independent modeling of the dilute solution conformation of the immunoglobulin IgG Fab' domain using SOLPRO and ELLIPS Biophys. J. 77 2902-10

[12] von Smoluchowski M 1916 Drei Vortrage uber Diffusion, Brownsche Bewegung und Koagulation von Kolloidteilchen Phys. Z. 17 557-71, 585-99

[13] Wilson I A and Stanfield R L 1993 Antibody-antigen interactions Curr. Opin. Struct. Biol. 3 113-8

[14] Traytak S D 1995 Diffusion-controlled reaction rate to an active site Chem. Phys. 192 1-7

[15] Barzykin A V and Shushin A I 2001 Effect of anisotropic reactivity on the rate of diffusion-controlled reactions: comparative analysis of the models of patches and hemisphere Biophys. J. 80 2062-73

[16] Janin J 1997 The kinetics of protein-protein recognition Proteins: Structure, Function and Genetics 28 153-61

[17] Piazza F, De Los Rios P, Fanelli D, Bongini L and Skoglund U 2005 Anticooperativity in diffusion-controlled reactions with pairs of partially absorbing domains: a model for the antigen-antibody encounter Eur. Biophys. J. 34 899-911 
[18] Carrasco B, De la Torre J G, Davis K G, Jones S, Athwal D, Walters C, Burton D R and Harding S 2001 Crystallohydrodynamics for solving the hydration problem for multi-domain proteins: open physiological conformations for human IgG Biophys. Chem. 93 181-96

[19] http://www.biacore.com/lifesciences/index.html

[20] Ligler F S and Rowe Taitt C A (ed) 2005 Optical Biosensor: Present and Future (Amsterdam: Elsevier)

[21] Malmborg A C, Micaelsson A, Ohlin M, Jansson B and Borrebaeck C A K 1992 Real-time analysis of antibody antigen reaction kinetics Scand. J. Immunol. 35 643-50

[22] George A J T, French R R and Glennie M J 1995 Measurement of kinetic binding constants of a panel of anti-saporin antibodies using a resonant mirror biosensor J. Immunol. Methods 183 51-63

[23] Kaufman E N and Jain R K 1992 Effect of bivalent interaction upon apparent antibody affinity: experimental confirmation of theory using fluorescence photobleaching and implications for antibody binding assays Cancer Res. 52 4157-67

[24] Berezhkovskii A M, Monine M I, Muratov C B and Shvartsman S Y 2006 Homogenization of boundary conditions for surfaces with regular arrays of traps J. Chem. Phys. 124036103

[25] Berezhkovskii A M, Makhnovskii Yu A, Monine M I, Zitserman V Y and Shvartsman S Y 2004 Boundary homogenization for trapping by patchy surfaces $J$. Chem. Phys. 121 11390-94

[26] Skoglund U, Ofverstedt L G, Burnett R M and Bricogne G 1996 Maximum-entropy three-dimensional reconstruction with deconvolution of the contrast transfer function: a test application with adenovirus J. Struct. Biol. 117 173-88

[27] Weiss S 2000 Measuring conformational dynamics of biomolecules by single molecule fluorescence spectroscopy Nat. Struct. Biol. 7 724-9

[28] Berdnikov V M and Doktorov A B 1981 The steric factor in the elementary act in the liquid phase Theor. Eksp. Khim. 17 318

[29] Temkin S I and Yakobson B I 1984 Diffusion-controlled reactions of chemically anisotropic molecules J. Phys. Chem. 88 2679-82

[30] Lee S and Karplus M 1987 Kinetics of diffusion-influenced bimolecular reactions in solution: II. Effects of the gating mode and orientation-dependent reactivity J. Chem. Phys. 86 1904-21

[31] Solc K and Stockmayer W H 1973 Kinetics of diffusion-controlled reaction between chemically asymmetric molecules. II. Approximate steady-state solution Int. Chem. Kinet. 5 733-52

[32] Berg O G 1985 Orientation constraints in diffusion-limited macromolecular association. The role of surface diffusion as a rate-enhancing mechanism Biophys. J. 47 1-14 